\title{
Les espèces du genre Tabebuia susceptibles de fournir le bois d'ipé
}

\section{Pierre Détienne}

Michel VernaY

Cirad

Upr Production et valorisation des bois tropicaux

73, rue Jean-François Breton 34398 Montpellier Cedex 5

France
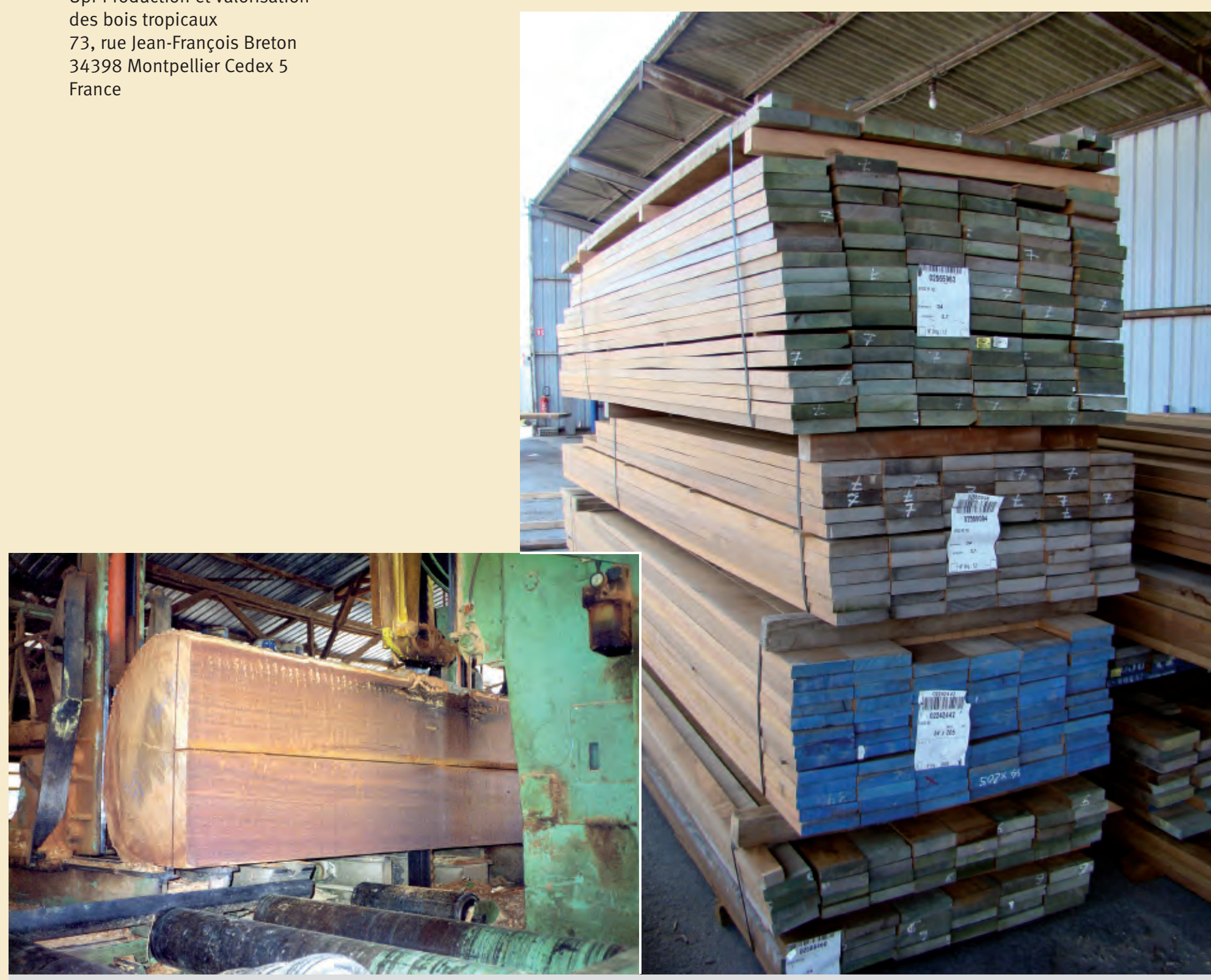

Photos 1.

Sciage d'une grume de Tabebuia serratifolia en Guyane et colis de débits d'ipé certifié FSC (Forest Stewardship Council) au port de Nantes. Photos M. Vernay. 


\section{RÉSUMÉ}

\section{LES ESPÈCES DU GENRE TABEBUIA SUSCEPTIBLES DE FOURNIR LE BOIS D'IPÉ}

Il s'avère que le prestigieux "bois d'ipé ", issu d'espèces du genre Tabebuia, domine le marché du matériau bois en extérieur haut de gamme depuis plusieurs années, ce qui est justifié par les remarquables propriétés physiques, mécaniques et de durabilité des espèces commercialisées sous cette appellation. Mais, du fait de l'engouement industriel pour ce type de matériau, l'offre sur le marché devient de plus en plus disparate avec la présence d'espèces ou d'essences n'ayant pas les propriétés attendues et recherchées. Cette note, basée sur l'ensemble du genre botanique Tabebuia, identifie les espèces dont les bois ont la capacité et les caractéristiques technologiques dévolues à l'appellation ipé. Une liste a été définie comprenant onze espèces du genre Tabebuia susceptibles de répondre aux exigences de qualité escomptées et de figurer ainsi sous l'appellation commerciale d'ipé. Quoi qu'il en soit, la dérive commerciale qui se fait jour pour l'ipé et ses ersatz représente une première alarme de raréfaction de la diversité biologique de certaines espèces du genre Tabebuia et demande que la plus grande prudence soit accordée aux risques de mélanges d'essences dans les lots commerciaux.

Mots-clés : genre Tabebuia, ipé, propriété du bois, utilisation du bois, marché du bois.

\section{ABSTRACT}

\section{POTENTIAL SOURCES OF IPÉ WOOD FROM SPECIES OF THE GENUS TABEBUIA}

“Ipé wood”, a prestigious timber variety from tree species of the genus Tabebuia, has dominated the market for high-quality exterior wood for a number of years, and justifiably so given the remarkable physical and mechanical properties and durability of the species marketed under that name. However, due to the popularity of the material among industrialists, supplies on the market are becoming increasingly disparate, with species being marketed whose sought-after properties do not meet expectations. In this research note, for which we studied the entire botanical genus Tabebuia, we identify the species whose wood has the technological capacities and characteristics attributed to what is known as Ipé wood. We provide a list of eleven Tabebuia species that can potentially satisfy the quality requirements expected of Ipé wood and can therefore be marketed under that name. In any event, the shift from true to ersatz Ipé wood now emerging on the market is a warning that the biodiversity of Tabebuia species is declining fast, which demands stringent precautions to avoid the risk of mixing species in commercial batches.

Keywords: genus Tabebuia, Ipé, properties of wood, uses of wood, market for wood.

\section{RESUMEN}

\section{ESPECIES DEL GÉNERO TABEBUIA CAPACES DE PROPORCIONAR MADERA DE IPÉ}

Es un hecho que la prestigiosa "madera de Ipé" procedente de especies del género Tabebuia, domina el mercado de materiales de madera de gama alta para exteriores desde hace varios años. Esto se debe a las notables propiedades físicas, mecánicas y de durabilidad de las especies comercializadas bajo esta denominación. Pero, debido al gran interés industrial que despierta este tipo de material, la oferta en el mercado se vuelve cada vez más dispar con la presencia de especies o variedades vegetales que no poseen las propiedades esperadas y buscadas. Esta nota, basada en el conjunto del género botánico Tabebuia, identifica aquellas especies cuya madera posee la capacidad y las características tecnológicas atribuidas a la denominación Ipé. Se estableció una lista que incluía once especies del género Tabebuia capaces de satisfacer las exigencias de calidad previstas, pudiendo así figurar bajo la denominación comercial de Ipé. De cualquier modo, la deriva comercial que se manifiesta en el Ipé y sus sucedáneos es la primera señal de alerta sobre la rarefacción de la diversidad biológica de ciertas especies del género Tabebuia y requiere que se aplique una extrema prudencia a los riesgos de mezclas de especies en los lotes comerciales.

Palabras clave: género Tabebuia, Ipé, propiedades de la madera, utilización de la madera, mercado de la madera. 


\section{Introduction}

Le bois d'ipé, produit par certaines espèces du genre botanique Tabebuia (famille des Bignoniacées), est très apprécié pour ses qualités qui en font un bois idéal pour de nombreuses utilisations tant en milieu protégé qu'à l'extérieur. Depuis quelques années, l'ipé domine avantageusement le marché de la lame de terrasse et des aménagements extérieurs haut de gamme. L'ipé concurrence les essences africaines et asiatiques moins performantes sur les aspects esthétiques, physiques, mécaniques, ou alors devenues plus rares et plus chères. Cette essence doit surtout sa notoriété à quelques propriétés physiques avantageuses : une forte densité qui en fait un bois très dur, une stabilité dimensionnelle remarquable due à ses faibles retraits au séchage et à son point de saturation des fibres très bas. L'ipé figure parmi les essences appartenant aux classes de résistance mécanique les plus fortes, selon la norme française NF EN 338 définissant ces classes pour les bois de structure. Son emploi peut donc être recherché pour des situations à fortes sollicitations mécaniques.

La forte demande en bois d'ipé a eu pour conséquence l'accroissement de l'hétérogénéité des lots commercialement proposés. Autrefois, l'ipé était essentiellement caractérisé par sa couleur très sombre et sa densité très élevée ; mais des bois à teinte relativement plus claire ou plus rougeâtre ou à densité relativement plus faible sont de plus en plus fréquemment rencontrés sur le marché. Cela signifie une probable raréfaction inquiétante des essences étant à même de produire du bois d'ipé. En excluant les cas où des bois de cumaru (Dipteryx spp., famille des Fabacées), de tanimbuca (Buchenavia spp. et Terminalia spp., famille des Combrétacées) ou de ita-uba (Mezilaurus spp., famille des Lauracées) peuvent se glisser dans certains lots, tout en tenant compte de la variabilité du bois à l'intérieur d'une espèce, il est certain que les bois de plusieurs espèces du genre Tabebuia, en plus de celles citées dans la nomenclature générale des bois tropicaux (AтіBT, 1982), sont commercialisés - parfois abusivement - sous le nom d'ipé. Quel est le nombre de ces espèces et quelles sont-elles ? Cette succincte note technique propose d'y répondre le plus exhaustivement possible, malgré le fait que le genre Tabebuia présent dans toutes les régions tropicales du Nouveau Monde soit relativement important par le nombre d'espèces. Dans la dernière révision de la famille des Bignoniacées américaines, le botaniste A. H. GENTRY (1992) recense exactement cent espèces, depuis Tabebuia acrophylla Britton jusqu'à Tabebuia zanonii A.H. Gentry, toutes produisant des arbres, arbustes ou arbrisseaux. Conséquence de son importance numérique, ce genre présente une certaine hétérogénéité, tout du moins pour des caractéristiques faciles à appréhender : la hauteur moyenne des sujets qui va de un mètre $(\mathrm{m})$ (Tabebuia pumila A.H. Gentry) à plus de $40 \mathrm{~m}$ (Tabebuia capitata Sandw. et autres), la couleur des fleurs, blanche ou avec diverses teintes de jaune ou de rouge, la couleur et la densité du bois de cœur, depuis le bois blanchâtre de densité 0,35-0,50 (à environ $12 \%$ d'humidité) jusqu'au bois brun-noir dont la densité dépasse 1,20.

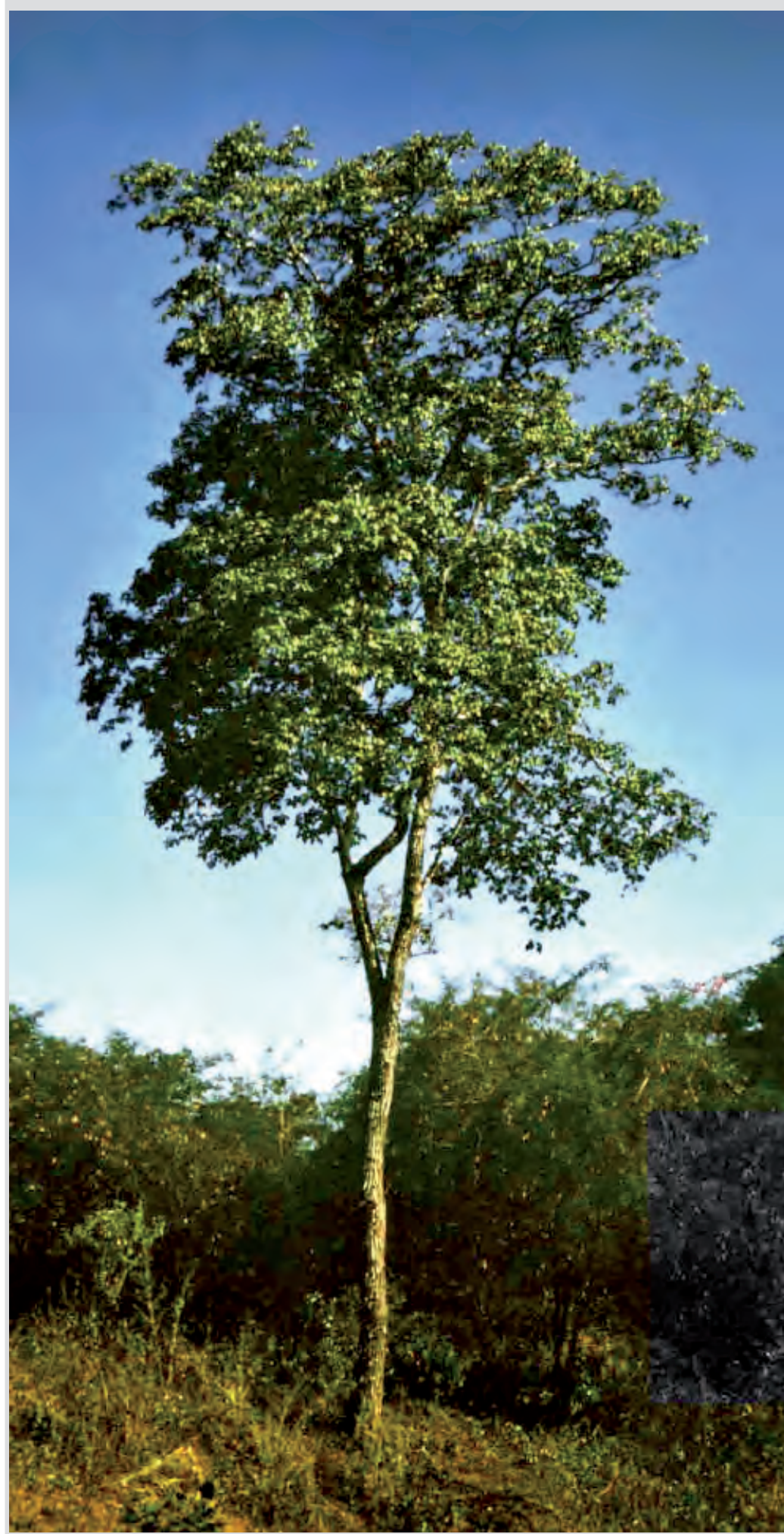

Photo 2.

Tabebuia billbergii Standl., dans la région d'Arenillas

(Équateur).

Photo M. Vernay. 


\section{Bref historique des espèces exploitées}

Il s'avère que, par le passé, les arbres du genre Tabebuia semblaient être commercialement peu connus, du fait de leurs variabilité ; mais aussi parce que leurs noms locaux souvent basés sur l'aspect de leur écorce (ipé signifie écorce en langue guarani) pouvaient aussi désigner des arbres appartenant à d'autres familles botaniques. Ainsi le nom tauari (ou tahuari) désigne-t-il des arbres dont la partie interne de l'écorce, légèrement battue, se délite en feuilles très minces (utilisables pour rouler des cigarettes). II s'applique à des espèces de Tabebuia comme à des espèces des genres Couratari, Eschweilera et Lecythis de la famille des Lécythidacées. De même, le nom de pau d'arco est donné à des espèces de Tabebuia ainsi qu'à des espèces des genres Dipteryx et Platymiscium de la famille des Fabacées.

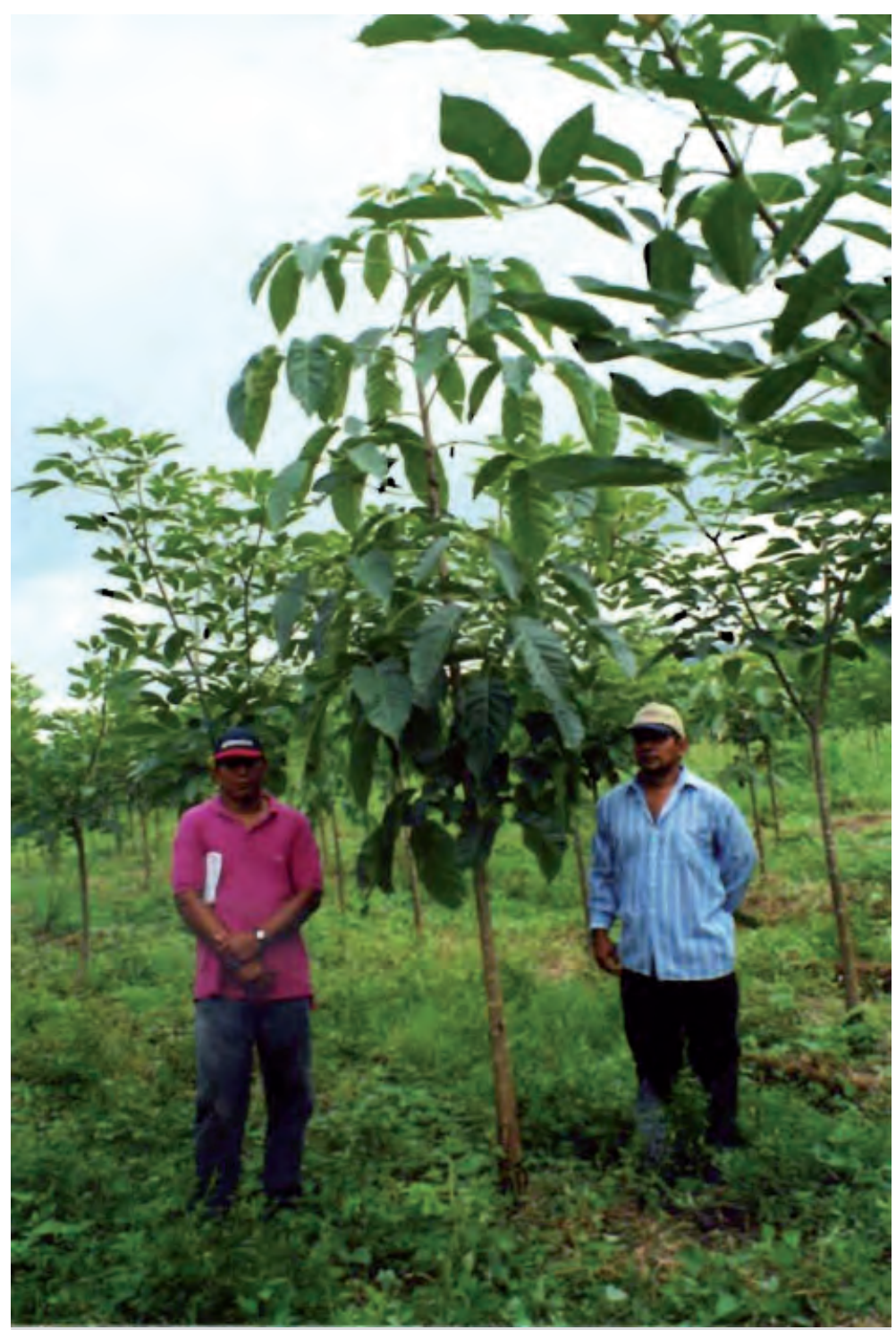

Photo 3.

Tabebuia rosea A.P.DC. (guayacán ou roble de Guayaquil). Jeunes plantations de 4 ans sur sols périodiquement inondés (Équateur). Photos H.-F. Maître.
En 1867, Freire AlLEMAO et al. signalent cinq essences pouvant appartenir au genre Tabebuia :

- caixeta, non identifié, poussant dans les États du Rio Grande do Sul, de Santa Catarina et de Rio de Janeiro ; cet arbre pourrait être rapproché de l'espèce Tabebuia cassinoides A.P.DC. ;

- ipé, Tecoma chrysantha A.P.DC. (aujourd'hui synonyme de Tabebuia chrysantha Nicholson), signalé comme étant un arbre de 11-13 m de hauteur dans le bassin amazonien; - ipé batata, Tecoma leucantha Fr. All. (?), dont le bois a les mêmes caractéristiques que celui de l'ipé ; il ne s'agit donc pas de Tabebuia leucantha Gomes, synonyme de l'actuel Tabebuia cassinoides A.P.DC., dont le bois est blanchâtre et léger ;

- ipé rana, Tecoma sp. (très probablement une espèce actuellement intégrée au genre Tabebuia), dans les États d'Amazonas et du Pará, produisant un bois rougeâtre préconisé seulement pour des menuiseries intérieures car moins résistant que l'ipé ;

- pau d'arco, Tecoma speciosa DC. (actuel Tabebuia serratifolia Nicholson), arbre de 20-30 m de hauteur, dans tout le bassin amazonien, dont le bois très résistant est utilisable pour des constructions civiles et navales (photos 1 ).

Dans son catalogue des plantes d'Amazonie publié en 1947, P. LE CoINTE signale les essences suivantes :

- capitari, Couroulia toxophora Benth. \& Hook.f. (Tabebuia barbata Sandw.), au bois brun sombre d'environ 1,03 de densité ; - carauba do campo, Tecoma caraiba Mart. (Tabebuia aurea Benth. \& Hook.), au bois blanc grisâtre, d'environ 0,71 de densité ;

- pau d'arco branco, Couroulia toxophora Benth. \& Hook.f. (Tabebuia barbata Sandw.) ;

- pau d'arco de flores amarelas, Tecoma conspicua DC. (Tabebuia serratifolia Nicholson), au bois brun sombre, très résistant, de 1,10 de densité ;

- pau d'arco de flores roxas, Tecoma violacea Huber. (GENTRY ne signale pas cette espèce, même comme synonyme) ; d'après les remarques de LE COINTE, " madeira semelhante à do Pau d'arco de flor amarela, muito dura, virando ao prêto com o tempo ", il s'agit très certainement de l'espèce actuelle Tabebuia impetiginosa Standl., seule espèce du genre, présente dans l'État du Pará, à avoir à la fois un bois sombre et lourd et des fleurs rougeâtres et non jaunes ; - tamura tuira, Tabebuia serratifolia Nicholson ;

- tauari, Tecoma aff. ochracea St. Hil. (Tabebuia aff. ochracea Standl.) à fleurs jaunes.

En principe, le premier classement du bois des espèces de Tabebuia a été proposé par RECORD et HESS (1943), avec quatre groupes d'espèces basés sur des différences d'aspect et de propriétés du bois :

- white cedar, regroupant Tabebuia aquatilis Sprague \& Sandw. (T. fluviatilis A.P.DC.) et T. insignis Sandw., donnant un bois beige-brun légèrement teinté de rose, d'une densité de $0,68-0,74$;

- roble, avec Tabebuia pentaphylla Hemsl. (actuellement divisé en $T$. rosea A.P.DC. et $T$. heterophylla Britton) et Tabebuia leptoneura Urb. au bois de couleur beige ayant une densité comprise entre 0,62 et 0,80 (photo 3). À ce groupe pourrait être adjointe l'espèce Cybistax donnell-smithii Seibert (Tabebuia donnell-smithii Rose) au bois identique ; 
- lapacho, rassemblant les espèces Tabebuia guayacan Hemsl., T. ipe Martius (T. heptaphylla Toledo), T. palmeri Rose (T. impetiginosa Standl.), T. rufescens J.R. Johnst. (T. chrysantha Nicholson), T. serratifolia Nicholson, auxquelles pourrait être ajoutée l'espèce $T$. barbata Sandw. ; toutes ces espèces étant caractérisées par leur bois de couleur brun-olive à noirâtre, dégageant une poussière jaune au sciage, ayant une densité comprise entre 0,95 et 1,25 ; - divers, composés des espèces Tabebuia cowellii Britton (T. trachycarpa K. Schum.), T. dubia Britton, T. lepidota Britton, T. maxonii Urban, T. mogotensis Urban (T. myrtifolia Britton), T. nodosa Gris., T. petrophylla Grenm. (T. myrtifolia Britton), $T$. schumanniana Urban et T. stenocalyx Sprague \& Sandw., toutes étant représentées par de petits arbres ou des arbustes.

Dans sa nomenclature générale des bois tropicaux de 1982, l'AтіBт retenait seulement cinq espèces groupées en trois essences commerciales :

- apamate, Tabebuia pallida Miers et T. rosea A.P.DC. ;

- ipé, Tabebuia ipe Martius (T. heptaphylla Toledo) et Tabebuia serratifolia Nicholson ;

- primavera, Tabebuia donnell-smithii Rose.

\section{Espèces susceptibles d’être exploitées}

Pour être exploitable afin de produire du bois d'œuvre en assez grande quantité, une espèce doit fournir des arbres atteignant et dépassant une hauteur de $20 \mathrm{~m}$. L'élimination de toutes les espèces dont les sujets n'atteignent pas $20 \mathrm{~m}$ réduit la liste des espèces proposées par GENTRY de 100 à 32 espèces. De plus, pour répondre aux exigences de production régulière de bois d'œuvre, les arbres d'une espèce doivent être relativement fréquents en forêt. S'il n'est pas possible de connaître la fréquence des arbres d'une espèce en s'appuyant sur de gigantesques inventaires forestiers qui ne peuvent pas être réalisés à l'échelle d'un continent, il est néanmoins admissible d'exclure les espèces présentes dans une seule île. C'est le cas, par exemple, de Tabebuia platyantha Britton à la Jamaïque, ou celles produisant de grands arbres mais dont l'aire est très restreinte, comme T. arianae A.H. Gentry, endémique à la vallée du Rio Doce dans l'État d'Espirito Santo au Brésil, ou T. coralibe Standl., endémique à la forêt semi-décidue au Nord de la Colombie. Il reste alors 21 espèces donnant de grands arbres d'au moins $20 \mathrm{~m}$ de hauteur supposés relativement fréquents sur le continent américain : Tabebuia alba Sandw., T. capitata Sandw., T. chrysantha Nicholson, T. chrysea Blake, T. donnell-smithii Rose, T. guayacan Hemsl., T. heptaphylla Toledo, T. heterophylla Britton, T. impetiginosa Standl., T. incana A.H. Gentry, T. insignis Sandw., T. ochracea Standl., T. pallida Miers, T. pedicellata A.H. Gentry, T. pulcherrima Sandw., T. rosea A.P.DC., T. roseo-alba Sandw., T. serratifolia Nicholson, T. stenocalyx Sprague \& Stapf, T. striata A.H. Gentry et T. uleana A.H. Gentry.

\section{Espèces susceptibles de fournir le bois d'ipé}

Les 21 espèces citées précédemment produisent des bois très divers et ce n'est que l'aspect du bois qui permet la sélection des espèces commercialement nommées ipé. D'après les observations faites sur les échantillons de Tabebuia des collections de l'US Forest Products Laboratory de Madison, SJRw et MADw (RECORD, HeSs, 1943 ; Dos SANTOS, Miller, 1992) et sur ceux de la collection du Cirad $^{1}$ (CTFw), les bois d'ipé doivent présenter simultanément les caractéristiques suivantes :

- densité supérieure à 0,85 ;

- couleur sombre, de beige-brun à brun rougeâtre ou brunnoir ; la présence de poudre jaune ou jaune verdâtre dans les pores est très typique mais il semble que des espèces n'en montrent pas, ou très peu, lors d'observations à l'œil nu ou avec l'aide d'une petite loupe (Tabebuia alba, T. capitata, T. chrysantha, T. pedicellata, T. pulcherrima, T. uleana) ;

- diamètre des ponctuations intervasculaires de 8-12-(14) $\mu \mathrm{m}$.

En excluant les espèces Tabebuia incana et $T$. striata, dont les bois n'ont pas été observés, les 11 espèces dont les bois répondent à ces critères sont les suivantes :

- Tabebuia alba Sandw. : arbres aux fleurs jaunes, atteignant $25 \mathrm{~m}$ de haut, mais généralement beaucoup plus petits, présents dans les zones subtropicales, du Sud du Minas Gerais (Brésil) jusqu'au Paraguay et au Nord de l'Argentine. - Tabebuia capitata Sandw. : arbres aux fleurs jaunes, de 40 m de haut, présents au Venezuela, dans les Guyanes, au Pérou et au Brésil (Acre, Rondônia, Amazonas, Roraima, Pará, Amapá, Maranhão).

- Tabebuia chrysantha Nicholson : arbres aux fleurs jaunes. Les sous-espèces chrysantha et meridionalis A.H. Gentry sont des arbres de taille moyenne, hauts de 10 à $20 \mathrm{~m}$, présents en Amérique centrale, en Colombie, au Venezuela, en Équateur et au Pérou. Les arbres de la sous-espèce pluvicola A.H. Gentry atteignent $30 \mathrm{~m}$ de haut au Costa Rica, au Panama, en Colombie, au Venezuela et en Équateur.

- Tabebuia guayacan Hemsl. : arbres aux fleurs jaunes, pouvant atteindre $50 \mathrm{~m}$ de haut, dans toute l'Amérique centrale, en Colombie, au Venezuela et au Pérou.

${ }^{1}$ Centre de coopération internationale en recherche agronomique pour le développement.

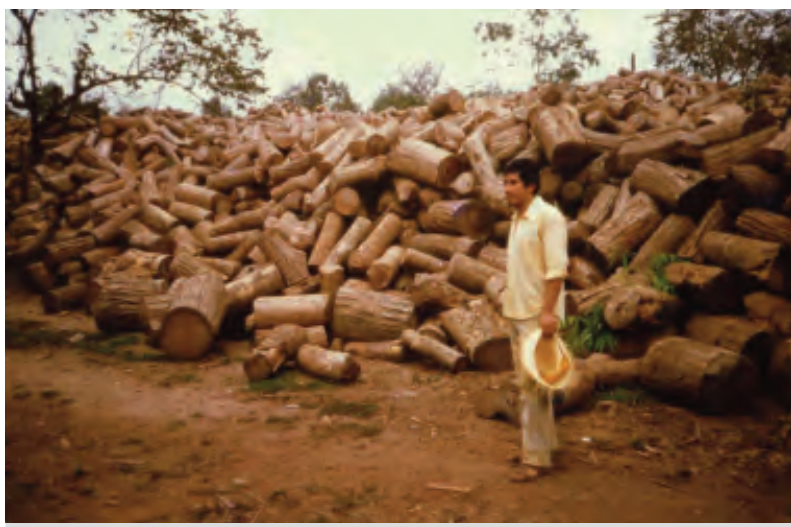

Photo 4.

Tabebuia billbergii Standl. débité en billons destinés à la fabrication de parquets haut de gamme (Équateur) Photo M. Vernay. 
- Tabebuia heptaphylla Toledo : arbres aux fleurs rouges magenta, variables en taille mais atteignant $30 \mathrm{~m}$ de haut, sur toute la bordure atlantique du Brésil (de l’État de Bahia jusqu'au Rio Grande do Sul), au Paraguay, au Nord de l'Argentine et en Bolivie.

- Tabebuia impetiginosa Standl. : arbres aux fleurs rouges magenta, de $30 \mathrm{~m}$ de haut, dans tous les pays du Mexique jusqu'au Sud du Brésil.

- Tabebuia ochracea Standl. : arbres aux fleurs jaunes. Les sousespèces ochracea (dans toutes les régions au Sud du fleuve Amazone) et heterotricha A.H. Gentry (en Colombie et au Venezuela) ne sont pas de grands arbres. Ceux de la sous-espèce neochrysantha A.H. Gentry atteignent 25 m, en Amérique centrale du Honduras au Panama, en Colombie et au Venezuela. - Tabebuia pedicellata A.H. Gentry : arbres aux fleurs jaunes, atteignant $20 \mathrm{~m}$ de haut, au Brésil dans les États du Minas Gerais, d’Espírito Santo et de Rio de Janeiro.

- Tabebuia pulcherrima Sandw. : arbres aux fleurs jaunes, atteignant 25 m de haut, au Sud du Brésil (Santa Catarina et Rio Grande do Sul), au Paraguay et au Nord de l'Argentine.

- Tabebuia serratifolia Nicholson : arbres aux fleurs jaunes, de $30 \mathrm{~m}$ de haut, sur tout le continent sud-américain au Nord d'une ligne Lima-São Paulo.

- Tabebuia uleana A.H. Gentry : arbres aux fleurs jaunes, de taille moyenne, atteignant $20 \mathrm{~m}$ de haut, en Colombie, au Venezuela, au Guyana et dans le Roraima (Brésil).
Il faut remarquer que ces 11 espèces retenues, ainsi que des espèces aux sujets de plus petite taille telles $T$. barbata Sandw. et T. billbergii Standl. (photos 2 et 4) ayant un bois lourd, de couleur sombre, avec des ponctuations intervasculaires grosses, appartiennent toutes aux groupes botaniques 3, 4 (fleurs jaunes, tube de la corolle glabre à l'extérieur) et 5 (fleurs rouges magenta, tube de la corolle pubérulent à l'extérieur) définis par A.H. GENTRY. Par extrapolation, il est permis d'imaginer que toutes les espèces appartenant à ces groupes 3, 4 et 5 - susceptibles de produire un bois lourd et de couleur brun sombre - seraient les suivantes : - Groupes 3 et 4 : T. alba Sandw., T. arianae A.H. Gentry, T. billbergii Standl., T. botelhensis A.H. Gentry, T. bureavii Sandw., T. capitata Sandw., T. catarinensis A.H. Gentry, T. chrysantha Nicholson, T. chrysotricha Standl., T. coralibe Standl., T. cristata A.H. Gentry, T. guayacan Hemsley, T. incana A.H. Gentry, T. lapacho Sandw., T. obscura Sandw., T. ochracea Standl., T. pedicellata A.H. Gentry, T. pulcherrima Sandw., T. pumila A.H. Gentry, T. riodocensis A.H. Gentry, T. serratifolia Nicholson, T. spongiosa Rizzini, T. subtilis Sprague \& Sandw., T. uleana A.H. Gentry, T. umbellata Sandw. et T. vellosoi Toledo.

- Groupe 5 : T. barbata Sandw., T. heptaphylla Toledo, T. impetiginosa Standl. et T. selachidentata A.H. Gentry.

\section{Encadré 1.}

Dénominations locales des principales espèces de Tabebuia produisant du bois d'ipé en Amérique du Sud.

ARAGUANEY (Venezuela), T. capitata, T. chrysantha, T. serratifolia ARAGUANEY ACAPRO (Venezuela), T. serratifolia

ARAGUANEY PUY (Venezuela), T. capitata, T. chrysantha

ARAWNIG ARUAIN (Guyana), T. serratifolia

ARUAIN (Guyana), T. serratifolia

CACHO VENADO (Venezuela), T. serratifolia

CANAGUATE (Venezuela), T. ochracea

CANAGUATE BLANCO (Venezuela), T. capitata, T. guayacan

CANAGUATE MORADO (Colombie), T. impetiginosa

CHACARADANGA (Venezuela), T. serratifolia

COMESEBO NEGRO (Pérou), T. capitata

ÉBÈNE VERTE (Guyane fr.), T. impetiginosa, T. serratifolia

GRIN-ATI (Surinam), T. serratifolia

GROENHART (Surinam), T. impetiginosa

GUAYACAN (Colombie), T. ochracea

GUAYACAN (Équateur), T. chrysantha,

GUAYACAN (Pérou), T. chrysantha

GUAYACAN (Venezuela), T. guayacan, T. ochracea

GUAYACAN DE LA COSTA (Équateur), T. chrysantha

GUINAATI (Guyane fr.), T. capitata, T. serratifolia

HAKIA ARUAIN (Guyana), T. serratifolia

IRONWOOD ARUAIN (Guyana), T. serratifolia

IPE (Argentine), T. heptaphylla

IPE (Brésil), T. alba, T. pulcherrima, T. serratifolia

IPE AMARELO (Brésil), T. alba, T. pedicellata, T. pulcherrima,

T. erratifolia

IPE BRANCO (Brésil), T. alba

IPE CABROE (Brésil), T. heptaphylla

IPE DA PRAIA (Brésil), T. pulcherrima

IPE DA SERRA (Brésil), T. alba

IPE DO CAMPO (Brésil), T. serratifolia
IPE MANDIOCA (Brésil), T. alba

IPE MAMONO (Brésil), T. alba

IPE OVO DE MACACO (Brésil), T. serratifolia

IPE PARDO (Brésil), T. alba, T. ochracea

IPE PRETO (Brésil), T. impetiginosa

IPE ROSA (Brésil), T. impetiginosa

IPE ROXO (Brésil), T. heptaphylla, T. impetiginosa

IPE UVA (Brésil), T. serratifolia

KONAWADRANUP ARUAIN (Guyana), T. serratifolia

KOONE (Surinam), T. serratifolia

LAPACHITO (Argentine), T. pulcherrima

LAPACHO (Argentine, Paraguay), T. alba, T. heptaphylla,

T. impetiginosa

LAPACHO AMARILLO (Argentine, Paraguay), T. alba, T. pulcherrima

LAPACHO CRESPO (Argentine), T. heptaphylla

LAPACHO MORADO (Argentine), T. heptaphylla

LAPACHO ROSADO (Argentine, Paraguay), T. impetiginosa

LAPACHO NEGRO (Argentine), T. heptaphylla

LAPACHO NEGRO (Paraguay), T. heptaphylla

MAKA-GRIN (Surinam), T. capitata

MAPACHILLO (Argentine), T. pulcherrima

PAU D’ARCO (Brésil), T. capitata, T. impetiginosa

PAU D'ARCO AMARELO (Brésil), T. alba, T. capitata, T. serratifolia

PAU D’ARCO PRETO (Brésil), T. capitata

PAU D'ARCO ROXO (Brésil), T. heptaphylla

PAU D'ARCO TATAJIPOCA (Brésil), T. capitata

POLVILLO (Colombie, Venezuela), T. impetiginosa

POROTILLO (Équateur), T. chrysantha

RANOI ARUAIN (Guyana), T. serratifolia

ROBLE MORADO (Colombie), T. impetiginosa

TAHUARI (Pérou), T. capitata, T. guayacan

TAIIY PICHAI (Argentine, Paraguay), T. impetiginosa

TAIIY ZAIYU (Paraguay), T. heptaphylla

VERO (Venezuela), T. ochracea, T. serratifolia 


\section{Caractéristiques des bois d'ipé}

Les bois des 11 espèces donnant de grands arbres présentent beaucoup de points communs dans leurs caractéristiques visibles à l'œil nu ou au microscope, et les variations constatées, à l'échelle macroscopique comme à l'échelle microscopique, semblent aussi importantes entre les échantillons d'une même espèce qu'entre les bois de deux espèces différentes.

La couleur du bois de cœur, très généralement sombre allant du brun olivâtre clair au brun rougeâtre et au brun noirâtre, ne permet pas de différencier efficacement une espèce ou un groupe d'espèces. Tout au plus, il semble que les bois aux teintes rougeâtres appartiennent plutôt aux espèces ayant peu ou pas de lapachol (T. alba, T. capitata, T. chrysantha, T. pedicellata, T. pulcherrima et T. uleana). Le lapachol se présente sous la forme d'une poudre jaune, identique au soufre, remplissant les pores et qui a la particularité de virer au rouge lorsque le bois est trempé dans une solution basique. Ce lapachol est généralement abondant et bien visible dans les pores du bois des espèces T. guayacan, T. heptaphylla, $T$. impetiginosa, $T$. ochracea et $T$. serratifolia.

La densité (à environ $12 \%$ d'humidité) est toujours comprise entre 0,85 et 1,30 . Elle ne permet pas non plus de différencier des espèces. Ainsi, les bois observés de l'espèce $T$. heptaphylla ayant une densité entre 0,87 et 1,09 sont en principe plus légers que ceux de l'espèce $T$. serratifolia ayant une densité de 1,00 à 1,26, mais sans en être efficacement séparables par ce critère.

Les débits rabotés sur quartier montrent toujours un contrefil serré (longueur d'onde de quelques millimètres à $1 \mathrm{~cm}$ ), moyen à fort, jusqu'à provoquer des arrachements lors du rabotage. L'intensité de ce contrefil semble être plus une caractéristique de l'arbre que de l'espèce.
Les débits rabotés sur dosses font apparaître l'étagement de la structure du bois, qui est matérialisé par l'alignement horizontal des rayons. Le nombre d'étages par millimètre est très souvent compris entre 3,5 et 4,5 , les espèces $T$. guayacan, T. impetiginosa et $T$. serratifolia montrant plutôt 3-4 étages par millimètre, et $T$. ochracea plutôt 4-5 étages par millimètre.

Le grain du bois, notion très subjective, ne permet pas de distinguer particulièrement une ou plusieurs espèces. En revanche, la fréquence des pores, plus que leur diamètre moyen, divise les bois de ces espèces en deux groupes. Ceux ayant de 10 à 25 pores par millimètre carré d'environ 80-120 $\mu \mathrm{m}$ de diamètre, T. capitata, T. chrysantha, T. guayacan, $T$. impetiginosa, $T$. serratifolia et $T$. uleana, et ceux ayant 30 à 70 pores par millimètre carré d'un diamètre de 60 à $100 \mu \mathrm{m}, T$. alba, T. heptaphylla, T. ochracea, T. pedicellata et $T$. pulcherrima. L'observation complémentaire du lapachol (dans le bois de cœur) permet de séparer assez grossièrement ces 11 espèces en quatre groupes (photo 5) : - Groupe 1, 10 à 25 pores par millimètre carré, souvent pleins de lapachol : T. guayacan, T. impetiginosa et $T$. serratifolia (T. guayacan ayant des ponctuations intervasculaires de 8-10 $\mu \mathrm{m}$, contre 10-12 $\mu \mathrm{m}$ chez les deux autres).

- Groupe 2, 10 à 25 pores par millimètre carré, contenant très peu ou pas de lapachol : T. capitata, T. chrysantha et $T$. uleana ( $T$. uleana ayant des ponctuations intervasculaires de $12 \mu \mathrm{m}$, contre 8-10 $\mu \mathrm{m}$ chez les deux autres).

- Groupe 3, 30 à 70 pores par millimètre carré, souvent pleins de lapachol : T. heptaphylla et $T$. ochracea ( $T$. ochracea ayant des ponctuations intervasculaires de 9-11 $\mu \mathrm{m}$, celles de T. heptaphylla étant plutôt de 11-13 $\mu \mathrm{m}$ ).

- Groupe 4 : 30 à 70 pores par millimètre carré, contenant très peu ou pas de lapachol : T. alba, T. pedicellata et T. pulcherrima ( $T$. alba ayant des ponctuations intervasculaires de 8-10 $\mu \mathrm{m}$, contre 10-12 $\mu \mathrm{m}$ chez les deux autres).
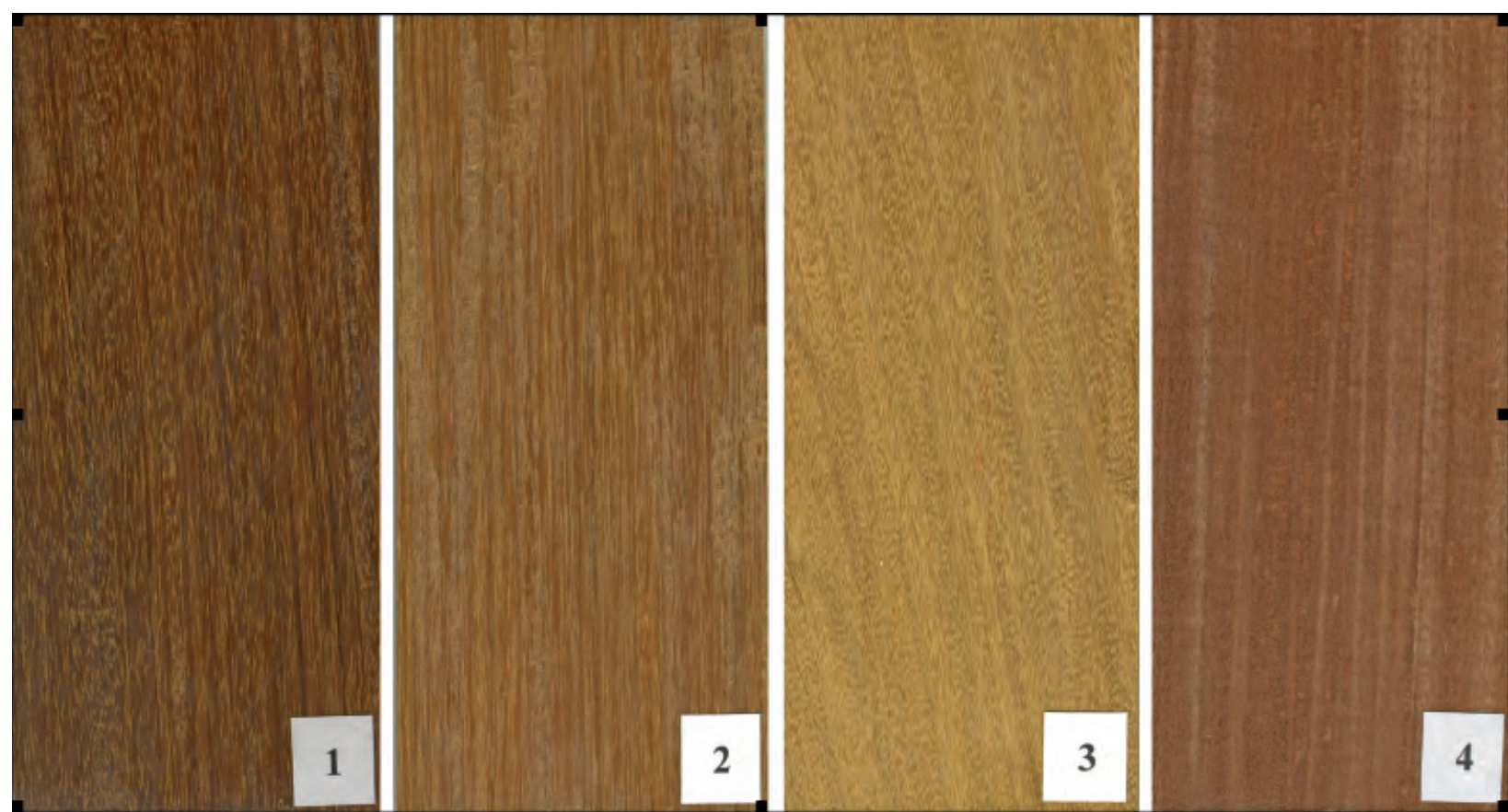

Photo 5.

Aspect du bois de : 1, Tabebuia serratifolia du Surinam ; 2, T. capitata du Brésil ; 3, T. heptaphylla du Paraguay ; 4, T. pedicellata du Brésil. 


\section{Incidence des caractéristiques des bois sur leur utilisation}

La densité, la couleur et la présence de contrefil sont les trois principaux critères facilement identifiables, responsables de l'hétérogénéité rencontrée dans les lots commerciaux d'ipé de ces 11 espèces.

\section{La densité}

La densité a une influence directe sur l'usinage (profilage et moulurage) et sur la mise en œuvre (facilité de perçage et de vissage).

Le bois d'ipé à $16 \%$ d'humidité (humidité moyenne d'équilibre du bois en service à l'extérieur en France) n'a pas systématiquement une densité supérieure à $1\left(1000 \mathrm{~kg} / \mathrm{m}^{3}\right)$. Une pièce de bois d'ipé immergée dans l'eau ne va donc pas obligatoirement « couler » mais peut très bien " flotter », ce qui vient contredire les idées reçues et souvent avancées comme argument commercial pour cette essence.

Le bois d'ipé a une résistance au poinçonnement et à l'usure par frottement qui est directement liée à sa densité. Les bois ayant la plus forte résistance au poinçonnement sont donc les plus denses. Le bois provenant d'ipé de faible densité est en général plus clair et moins nuancé que celui des espèces les plus denses.

\section{La couleur}

La couleur est une caractéristique qui doit être prise en compte pour certains emplois. La variabilité importante de couleur peut nuire à l'esthétique de certains ouvrages, en particulier en ébénisterie, en parqueterie, ou dans les emplois sous forme de parement et en décoration.

Pour des ouvrages extérieurs soumis aux intempéries, où aucun entretien spécifique n'est prévu, le mélange des couleurs a peu d'importance. Le bois d'ipé subit un « grisaillement » naturel assez homogène se rapprochant du grisbrun foncé lorsque le bois est mouillé et du gris argenté lorsque la surface du bois est sèche.

\section{Le fil du bois}

Le fil du bois d'ipé est généralement droit mais la présence de contrefil sur le bois de certains arbres est possible. Lorsque ce contrefil est régulier, il apporte un « rubanage » esthétique au bois. Un contrefil irrégulier et/ou fortement accusé rend le bois difficile à usiner. Son aspect de surface présente des arrachements et des soulèvements de fibres. Certains arbres dont le bois a un fil régulièrement sinueux dans le plan perpendiculaire à celui du contrefil présentent un intérêt pour le tranchage et le placage décoratif (bois à l'aspect moiré).

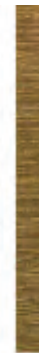
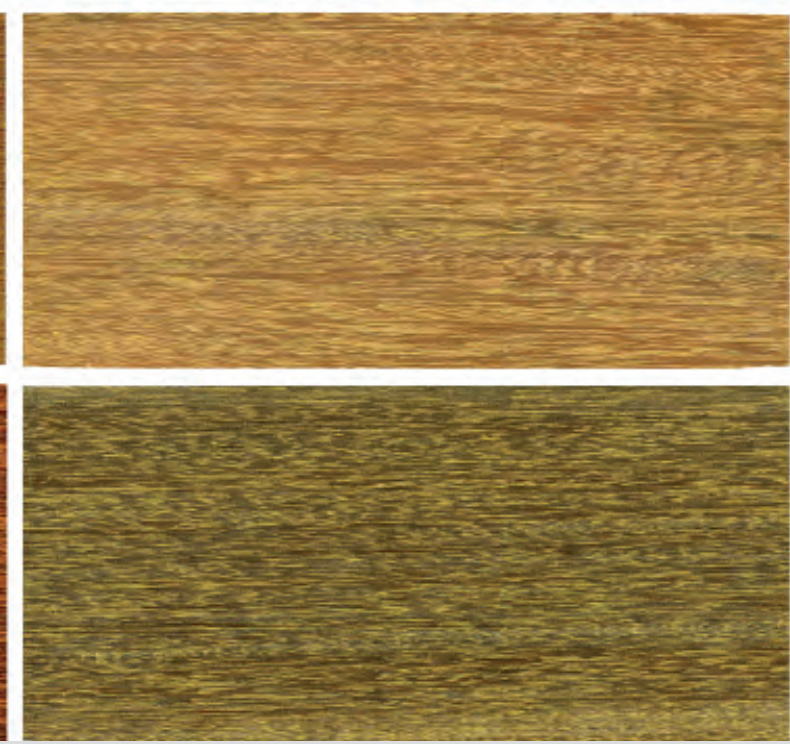
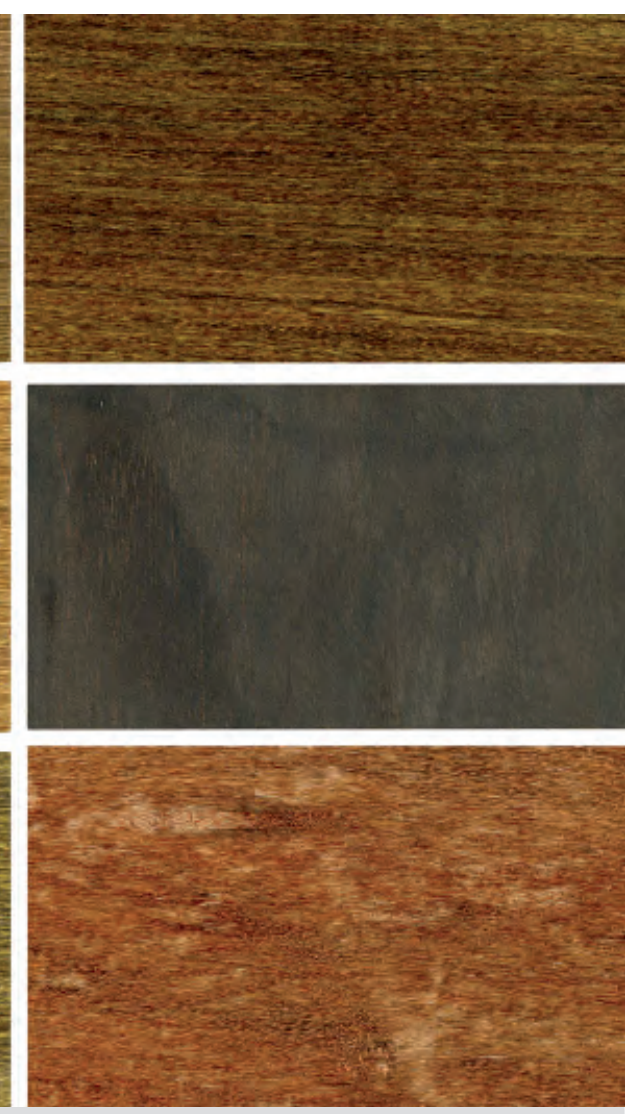

Photo 6.

Différentes couleurs de bois d'ipé. 


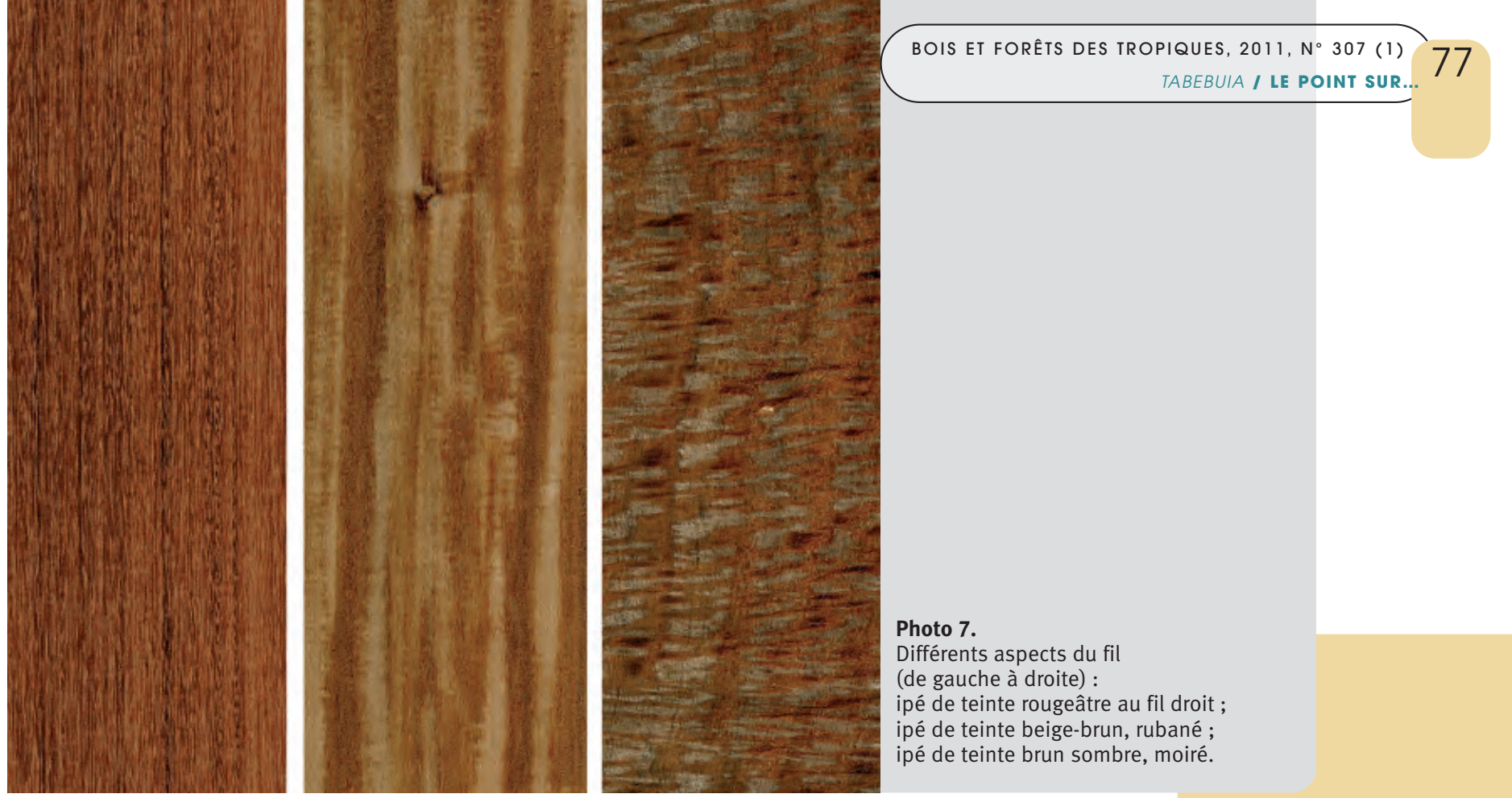

\section{Conclusion}

Depuis son arrivée sur le marché européen dans les années 1990, l'ipé, du genre botanique Tabebuia (famille des Bignoniacées), est considéré comme l'une des essences les plus nobles pour une utilisation dans des emplois extérieurs, principalement dans le secteur de la terrasse en bois. Ce secteur d'activité est devenu une véritable niche commerciale, obligeant même les prescripteurs et utilisateurs français à élaborer et à adopter en décembre 2010 un document technique unifié (Dtu) pour la conception et la réalisation des terrasses en bois. Ce document est accompagné d'une norme française définissant les caractéristiques exigées pour la réalisation de lames de platelage.

La demande en ipé sur le marché international n'a fait que croître au cours de la dernière décennie. La mise en place d'une exploitation forestière raisonnée et la forte pression commerciale sur une essence qui se raréfie ont provoqué une envolée des prix de ce bois. Le prix au mètre cube a été multiplié par six au cours des six dernières années.

La tentation est donc forte, de la part des opérateurs, d'étoffer ce créneau commercial en élargissant la gamme des produits pouvant porter le nom d'ipé. Outre le mélange avec des essences de même origine géographique (cumaru, tanimbuca, itauba...), les opérateurs n'hésitent pas à rechercher et à mettre sur le marché des produits issus du genre botanique Tabebuia dont les caractéristiques ne correspondent pas à celles qui sont attendues du bois d'ipé.

Une simple analyse de l'aspect et de la structure anatomique du bois permet, d'une part, de reconnaître son appartenance au genre Tabebuia et, d'autre part, de le ranger dans le groupe commercial approprié. Cette mise au point est largement justifiée compte tenu des dérives commerciales régulières qui sont constatées sur le marché des lames de platelage et des revêtements bois (decking).

\section{Références bibliographiques}

ATIBT, 1982. Nomenclature générale des bois tropicaux. $6^{\mathrm{e}}$ édition. Paris, France, Association technique internationale des bois tropicaux, $216 \mathrm{p}$.

DÉTIENNE P., JACQUET J., 1983. Atlas d'identification des bois de l'Amazonie et des régions voisines. Nogent-surMarne, France, Centre technique forestier tropical, 640 p.

DOS SANTOS G., MILLER R. B., 1992. Wood anatomy of Tecomae. In: Gentry A. H. (éd.). Flora Neotropica 25: Bignoniaceae. Part 2 (Tribu Tecomeae). New York, USA, New York Botanical Garden, p. 336-359.

FREIRE ALLEMAO F. F., ALVES SERRAO E., NETTO L., DE SALDANHA DA GAMA J., 1867. Breve noticia sobre a collecao das madeiras do Brasil. Rio de Janeiro, Brésil, Typographia nacional. GENTRY A. H., 1992. Flora Neotropica 25: Bignoniaceae. Part 2. New York, USA, New York Botanical Garden, 358 p.

LE COINTE P., 1947. Amazônia Brasileira. III. Arvores e plantas uteis. Rio de Janeiro, Brésil, Compagnia Editora Nacional, Biblioteca Pedagôdica Brasileira, coll. Brasiliana, série 5, vol. 251, 506 p.

RECORD S. J., HESS R. W., 1943. Timbers of the New World. New Haven, USA, Yale University Press, 640 p. 